\title{
Spectral Spreading Signals for Asynchronous CDMA
}

\author{
E. Del Re, R. Fantacci, L. S. Ronga \\ LENST - Dipartimento Ingegneria Elettronica - Via di Santa Marta 3 - 50139 FIRENZE - ITALY \\ Ph.+39-55-4796485 - Fax +39-55-4796485 - e-mail: \{ronga,delre,fantacci\}@lenst.die.unifi.it
}

\begin{abstract}
In asynchronous environments, the spreading signature generation is one of the main issues for CDMA communication systems. In this paper is shown that the spectral design of the spreading signature leads to efficient bandlimited signals with attractive cross-correlation properties. The behavior of the proposed spreading signals is simulated in a GSM-like multipath environment and it is compared to that of a traditional DS-CDMA using Walsh binary sequences.
\end{abstract}

Technical Subject Category: CDMA

\section{Introduction}

The DS-CDMA technique relies on an efficient design of the spreading codes, with a small cross-correlation index to permit the retrieval of the desired user's information, and an autocorrelation shape as closer as possible to the Dirac's delta; this to avoid unexpected auto-interference effects due to the reception of delayed version of the transmitted signal.

Sometimes the standard design in the time domain of the spreading codes may result inappropriate, especially in presence of multi-path where time-shifted replicas of the same signal interfere with the desired one.

The basic theory of the Digital Signal Processing suggests an alternative method to design spreading functions and the resulting technique has some interesting properties which may result useful in the presence of multi-path environments.

In this work we show how the spreading sequences can be alternatively designed in the frequency domain. The flat spectral occupancy, the auto-correlation and other interesting properties are highlighted, and some examples of applications are given in order to compare this design technique with the standard one.

The paper is organized as follows: after a short introduction in section I, the definitions of the spectral spreading functions is in section II. In section III is reported a comparison between the proposed signals and the traditional Direct Sequences, while in section IV the computer simulations are described and the corresponding results commented.

\section{Spectral Spreading Signals}

A complex representation of the spreading signals is considered. We can write the definition of the signals as fol- lows:

$$
s_{i}(t)=\sqrt{\frac{2}{T_{b}}} \sum_{k=1}^{K} S_{i}(k) e^{j 2 \pi k t / T_{b}}
$$

Where $S_{i}(k)$ is a complex-valued spectral coefficient. The signals $s_{i}(t)$ expressed by (1) are band-limited by $K / T_{b}$ since they are generated by a sum of harmonics whose higher frequency is $K / T_{b}$. The definition of $s_{i}(t)$ is due to the choice of a complex sequence of length $K$. We refers to (1) as spectral spreading signals.

In order to generate signals with flat amplitude spectrum and unitary energy, the values of $S_{i}(k)$ are chosen with constant modulus and variable phase, as follows:

$$
S_{i}(k)=\frac{1}{\sqrt{K}} e^{j \theta_{i}(k)} \quad \forall k, \forall i
$$

A polyphase or $\mathrm{N}$-Phase sequence is a sequence whose elements are of the form $\exp (j 2 \pi n / N)$, with $0 \leq n<N$.

If $r(x)$ and $q(x)$ are two N-Phase sequences of length $\mathrm{L}$, the Periodic Cross-correlation Function (PCCF) is defined as:

$$
\varphi_{r q}(l)=\sum_{x=0}^{L-1} r^{*}(x) q((x+l) \quad \bmod L) \quad 0 \leq l<L
$$

The respective autocorrelation function is obtained if the sequence $r(x)$ is replaced by $q(x)$.

The sequence $r(x)$ is called perfect if the side-lobes of the corresponding autocorrelation function are 0 .

There are some operations that, if applied to a perfect $\mathrm{N}$ Phase sequence, permit to generate further perfect N-Phase sequences of the same size. The invariance operations are:

1. reflections: $g(x)=r(-x)$

2. cyclic shifts: $g(x)=r(x-u)$ with $u$ integer

3. constant phase addition: $g(x)=r(x) * \exp (j 2 \pi u / N)$ with $u$ integer

4. linear phase addition: $g(x)=r(x) * \exp (j 2 \pi u x / N)$ with $u$ integer

5. proper decimation of rows: $g(x)=r(u x)$ where $u$ and $L$ are coprime.

Where each argument is intended modulus $L$. 
In the past a large number of methods have been developed for searching or generating perfect N-Phase sequences. The basic theory of perfect sequences generation is due to Frank, Chu and Milewski [3, 2, 4]. Synthesis methods able to generate new sequences of various length from the basic ones, are also known [1].

The interest in this class of multi-phase sequences derives from the expression of the cross-correlation factor between two spectral spreading signals defined by (1). In facts we have:

$$
\begin{aligned}
\left\langle s_{i}(t), s_{j}(t)\right\rangle & =\int_{-T_{b} / 2}^{T_{b} / 2} s_{i}(t) s_{j}(t) d t \\
& =\sum_{k=1}^{K}\left(a_{i}(k) a_{j}(k)+b_{i}(k) b_{j}(k)\right) \\
& =\operatorname{Re}\left\{\sum_{k=1}^{K} S_{i}^{*}(k) S_{j}(k)\right\}
\end{aligned}
$$

Equation (4) shows that the cross-correlation between two different signals is the real part of the complex crosscorrelation between the two sequences of the respective spectral coefficients. This suggests that the perfect sequences can be used as the spectral coefficients in spectral spreading signal definition.

We have focused our attention on the so called perfect arrays, whose definition follows:

$$
\begin{aligned}
& s(x, y)=\exp \left\{j \frac{2 \pi}{N}(x \cdot y)\right\} \\
& \quad 0 \leq x<N, \quad 0 \leq y<N
\end{aligned}
$$

In this case we are able to generate $\mathrm{N}$ different sequences of length $\mathrm{N}$. It can be easily shown that each sequence is perfectly orthogonal to any other. The number of codes generated equals the spreading factor, as in the case of direct sequences derived from the Walsh's matrix.

We analyze the behavior of the spectral spreading signals when the signals are received with unexpected delays. Since a synchronization device is supposed to be present, we consider the receiver perfectly synchronized with the main incoming signal of the desired user. By doing that in general the receiver is not synchronized with the interferers and all the delayed replicas of the desired signal due to multipath.

Under these conditions, the ideal behavior of a set of spreading signals $\hat{s}_{i}(t)$ is:

$$
\begin{aligned}
& \operatorname{Re}\left[\frac{1}{T_{b}} \int_{-T_{b} / 2}^{T_{b} / 2} \hat{s}_{i}^{*}(t-\tau) \hat{s}_{i}(t) d t\right]= \begin{cases}1 & \tau=t \\
0 & \text { otherwise }\end{cases} \\
& \operatorname{Re}\left[\frac{1}{T_{b}} \int_{-T_{b} / 2}^{T_{b} / 2} \hat{s}_{i}^{*}(t-\tau) \hat{s}_{j}(t) d t\right]=0 \quad i \neq j
\end{aligned}
$$

with $\tau$ ranging in $\left[-T_{b} / 2, T_{b} / 2\right]$.

\section{Comparison between direct sequences signals and spectral spreading signals}

We now consider a practical case and compare the auto/cross-correlation profiles of both direct sequences and perfect array phase sequences signals. We consider the situation where the same information symbol is continuously repeated by both the desired and the interferent user.

No phase distortions effects are taken into account and a spreading factor $K$ of 32 is considered.

Fig 1 shows the cross-correlation profile (continuous line) and the auto-correlation profile (dotted line) of two spreading signals.

In the upper part of the fig 1 , two signals generated by the proposed technique are compared. The upper-left part shows the effect of delay on two signals derived by Chu Perfect Sequences. Chu Sequences do not guarantee the perfect orthogonality with $r=0$, but the delayed crosscorrelation is bounded in the whole range of delay.

On the upper-right part is shown the profile for two signals derived by Perfect Arrays. This time the orthogonality is perfect for the synchronous case $(\tau=0)$ and the delayed profile is extremely low, with the exception of a narrow pulse. Basically the spreading signals derived from a Perfect Array are composed by a discrete cyclic delayed copy of the first sequence.

This may appear as an undesired property, but the narrowness of the impulse is the key issue here. As confirmed by computer simulations, later described, the cross correlation impulse is so narrow that only a few times a multipath replica is likely to fall inside it. This results in a global averaged good rejection of the delayed replicas of the interference signals.
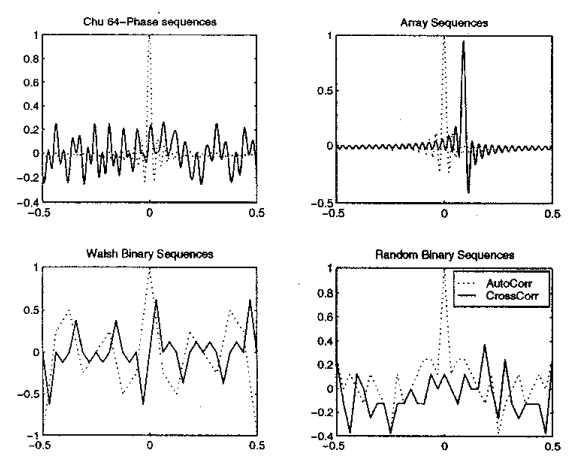

Figure 1: Auto- and cross-correlation profile in the asynchronous case

As the lower half of the fig 1 concerns, the same cross/auto correlation profile is computed for an ordinary Direct Sequences spreading signal. Each chip modulates a square pulse and both Walsh and random binary codes are represented. While orthogonality holds in the synchronous case also for Walsh DS codes, as soon as the signal is delayed, DS signals manifest poor cross and auto correlation profiles. 


\section{Computer Simulations and Results}

To prove the observations stated in the previous sections, several computer simulations have been made.

The general assumptions in common to each simulated environment are:

1. Two users sharing the same channel. One is the desired user whose information is wanted; the other one is the interferer.

2. AWGN noise added to the received signal,

3. Severe multipath channel,

4. Performance measured in terms of probability of error at the receiver for the desired user computed for different values of signal to noise ratio.

The CDMA system under test are:

- Direct Sequences CDMA with Walsh codes and rectangular pulse modulated by each chip,

- Phase Sequences CDMA whose complex codes are obtained by the perfect arrays.

We now prove that the unusual cross-correlation profile exerted by the perfect arrays in the CDMA using the proposed signaling is convenient in the presence of strong multipath. Starting from the GSM channel model, we have developed a discrete simulation environment capable to generate the effect of a strong multipath.

The channel model used in this set of simulations is basically a time-varying complex filter, whose taps are synchronously updated by several pink Rayleigh discrete processes.

The Fig. 2 helps to understand the structure of the channel model. The stream of samples is filtered by a complex Finite Impulse Response filter (fig. 3) whose dimension (the parameter WINDOW) influences the multipath spread of the channel. A white Gaussian complex process is then added to the resulting signal.

The taps of the complex filter are continuously updated by the Rayleigh generator and spread profiler block.

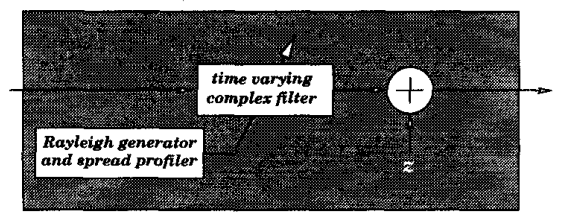

Figure 2: Discrete multipath channel model

Each tap of the complex filter is synchronously updated from a set of independent Rayleigh generators. The structure of each complex tap generator is described in the fig. 4 .

Each complex tap is generated by a Rayleigh process with unitary variance. This process is then filtered by a low pass FIR used to shape the power spectral density of

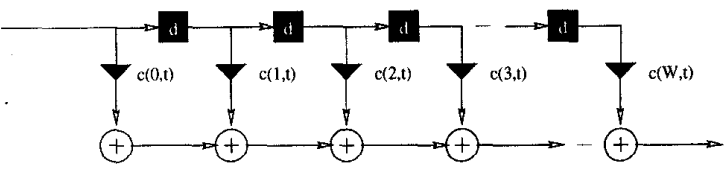

Figure 3: Time varying complex filter

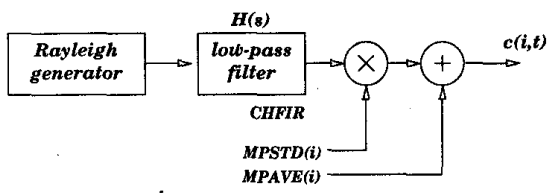

Figure 4: Complex Taps Generation

the process. The FIR taps are defined in the CHFIR vector which is a parameter of the simulation. Basically the CHFIR vector defines the frequency response of the spectral shaping filter $H(f)$. Since the Rayleigh generator is white, the quantity $\|H(f)\|^{2}$ gives the power spectral density of the channel taps. The first and second order statistics of the channel taps are defined separately for each tap by the two vectors MPAVE and MPSTD. Basically the tap $c(i, t)$ is a pink Rayleigh (or Rice) process with variance $M P S T D(i)^{2}$ and mean $M P A V E(i)$.

The two system, DS-CDMA and spectral spreading signals, have been tested with different values of the received interferer power. Table 1 shows the scalar parameters used in the simulation, while in the figs. 5 and 6 are plotted the values of the vectors involved.

\begin{tabular}{cl}
\hline spreading factor & 16 \\
\hline received user power & 1 \\
\hline received interferer power & $10,4.65,2.15$ \\
\hline SNR (desired user) dB & $0 \cdots 8$ \\
\hline FFT size & 32 \\
\hline WINDOW & 32 \\
\hline phase sequences type & perfect 32-phase arrays \\
\hline direct sequences type & Walsh 16 \\
\hline
\end{tabular}

Table 1: Asynchronous case - profile A - parameters

In the fig. 7 there is a comparison between a DS-CDMA system and the proposed one, both facing a severe multipath environment. The performance of the proposed technique is clearly better than the direct sequences. As expected this gap increases as the AWGN noise is reduced. The cross-correlation properties of the Phase sequences are useless when the dominant cause of error is the AWGN noise.

\section{Conclusions}

The design of spreading signatures for CDMA systems offers a better cross-correlation profile in the asynchronous environment with respect to the traditional direct sequences. An IFFT implementation of the signal genera- 


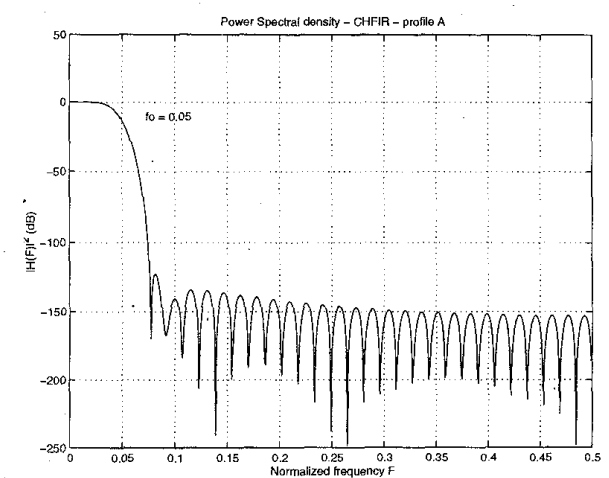

Figure 5: Profile A - vector parameters I

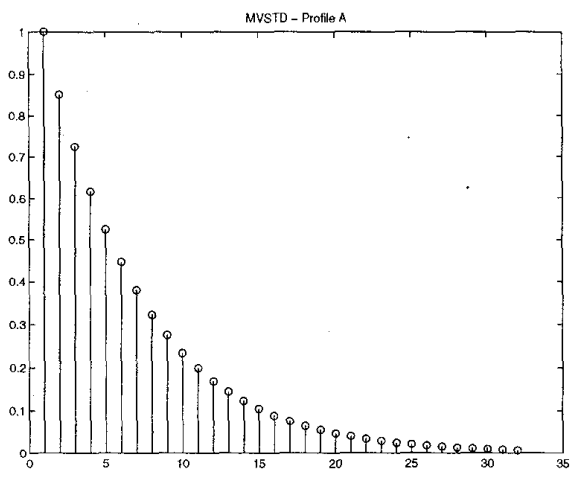

Figure 6: Profile A - vector parameters II

tor is possible, so that the resulting spreading technique fits the requirements for a cheap DSP realization in portable devices. The good average behavior in a multipath environment of the proposed signatures is confirmed by computer simulations.

\section{References}

[1] L. Bömer and M. Antweiler. Perfect n-phase sequences. IEEE Journal on Selected Areas in Commu nications, 10(4):782-789, May 1992.

[2] D. C. Chu. Polyphase codes with good periodic correlation properties. IEEE Trans. Inform. Theory, IT18:531-532, 1972.

[3] R. L. Frank and S. Zadoff. Phase shift pulse code with good periodic correlation properties. IEEE Trans. Inform. Theory, IT-8:381-382, 1962.

[4] A. Milewski. Periodic sequences with optimal properties for channel estimation and fast start-up equalization. IBM J. Res. Develop., 27:426-431, 1983.

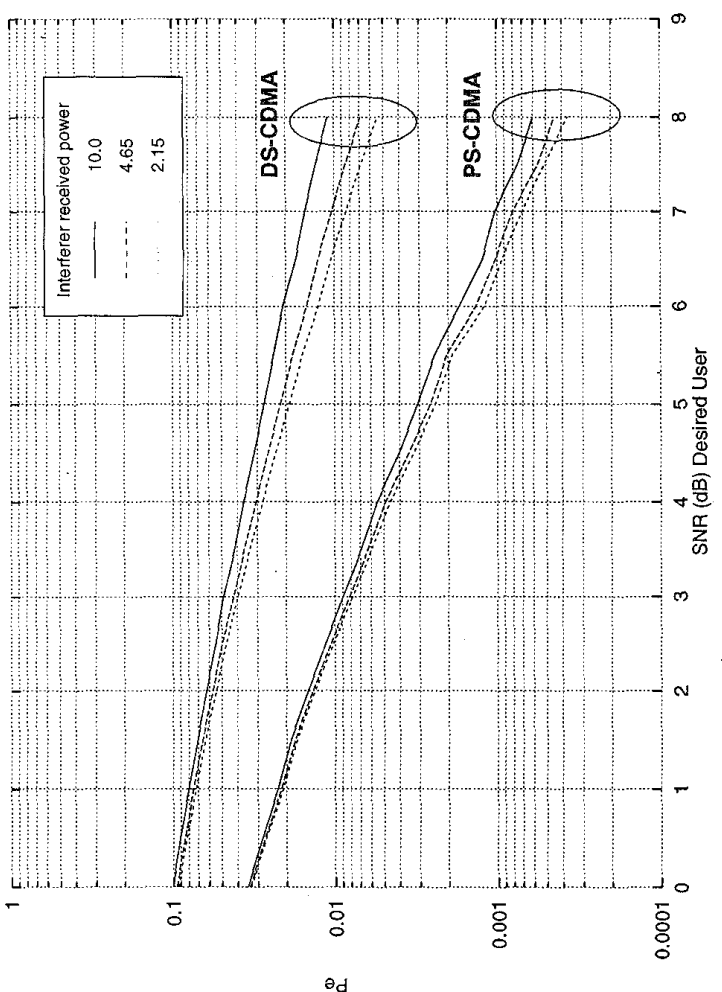

Figure 7: spectral spreading signal-CDMA and DS-CDMA in the asynchronous case 